\title{
Influence of Ionospheric Conditions on the Accuracy of High Frequency Direction Finding ${ }^{1,2}$
}

\author{
P. J. D. Gething
}

(August 22, 1960)

\begin{abstract}
The accuracy of fixes obtained by HF direction finding stations has been examined by means of a dispersion factor computed for each fix; this factor is a measure of the consistency of bearings taken from different stations on the same transmission. It is shown that the accuracy is significantly lower during times of ionospheric storms than at times when no storm occurred, and that the effect of the storm is mainly on fixes involving $F 2$ layer propagation.
\end{abstract}

\section{Introduction}

It is natural to suppose that the accuracy of DF bearings taken on transmissions in the $\mathrm{HF}$ band will depend to some extent on prevailing ionospheric conditions. In particular, ionospheric storms might be expected to lead to some deterioration of accuracy. The present paper describes a statistical analysis undertaken to test this hypothesis.

The bearings analyzed were obtained from stations equipped with standard Adcock direction finders. They were taken on both fixed and mobile transmitters, the majority of the transmitters being in the distance range 1,000 to $3,000 \mathrm{~km}$ from the DF stations. The true positions of the transmitters were not known in the majority of cases.

The quality of each bearing was estimated by means of the Brooke system. In this system, a variance is assigned to each bearing depending on several factors known to be related to accuracy. The variance components were originally estimated from an analysis of the errors in some thousands of bearings obtained on targets of known position; they are regularly revised on the basis of analysis of current data on such targets. The system is described in detail in an accompanying paper by E. M. L. Beale. It should be noted that the Brooke variances do not take account of variations in ionospheric conditions explicitly, although the components depending on the swing and range of snap bearings are probably correlated with such variations to some extent.

The bearings obtained on a given task from different DF stations do not normally intersect in a point. An electronic computer has been programed to solve the statistical problem of finding the most probable position of the transmitter (Best Point Estimate or BPE) and a surrounding rectangle representing a 90 percent probability region. The Brooke variances are used in the calculation, and affect the position of the BPE and the size and position of the rectangle. The computer also calculates

1 Contribution from Government Communications Headquarters, Cheltenham, England.

2 Paper presented at the Conference on Transmission Problems Related to High-Frequeney Direction Finding, at UCLA, June 21-24, 1960. a so-called 'dispersion factor,' the definition of which is given in section 3 . This factor is a measure of the consistency of three or more bearings taken from different stations on the same task. The average value of the dispersion factor should be $(n-2)$, where $n$ is the number of bearings. Under adverse conditions the average value would be greater than $(n-2)$.

The average value of dispersion factor has been used throughout the analysis as a measure of accuracy. The advantage of using this factor in a quantitative investigation is that it can be calculated on all tasks without any knowledge of the true positions of the transmitters.

\section{Definition of an Ionospheric Storm}

Various phenomena are associated with ionospheric storms, the most characteristic being the change in the critical frequency of the $F 2$ layer $\left(f_{0} F 2\right.$ ). The critical frequency often shows a brief initial rise, termed the positive phase, followed by a steep fall to a much longer negative phase, during which fo $F 2$ is below the value expected for calm conditions.

It is conventional to classify storms as moderate or severe. A moderate storm is defined in the B.B.C. monthly propagation reports as one in which fo $F 2$ lies between 60 percent and 90 percent of the fortnightly running hourly mean fo $F 2$, and a severe storm as one in which fo $F 2$ is less than 60 percent of this mean. The B.B.C. reports list days on which severe and moderate storms occurred.

The B.B.C. classification was used in the initial stages of the investigation. Later, in order to determine the magnitude and duration of each storm more precisely, published data from the U.K. vertical sounding stations at Slough and Inverness were examined. The start of the storm was then taken to be the time at which $f_{0} F 2$, measured at Slough, fell below 90 percent of the monthly median for the corresponding time. The storm became severe when fo $F 2$ fell below 60 percent of the same median value. The progress of a typical storm is shown in figure 1, where the Slough value of fo $F 2$ is plotted against time. 


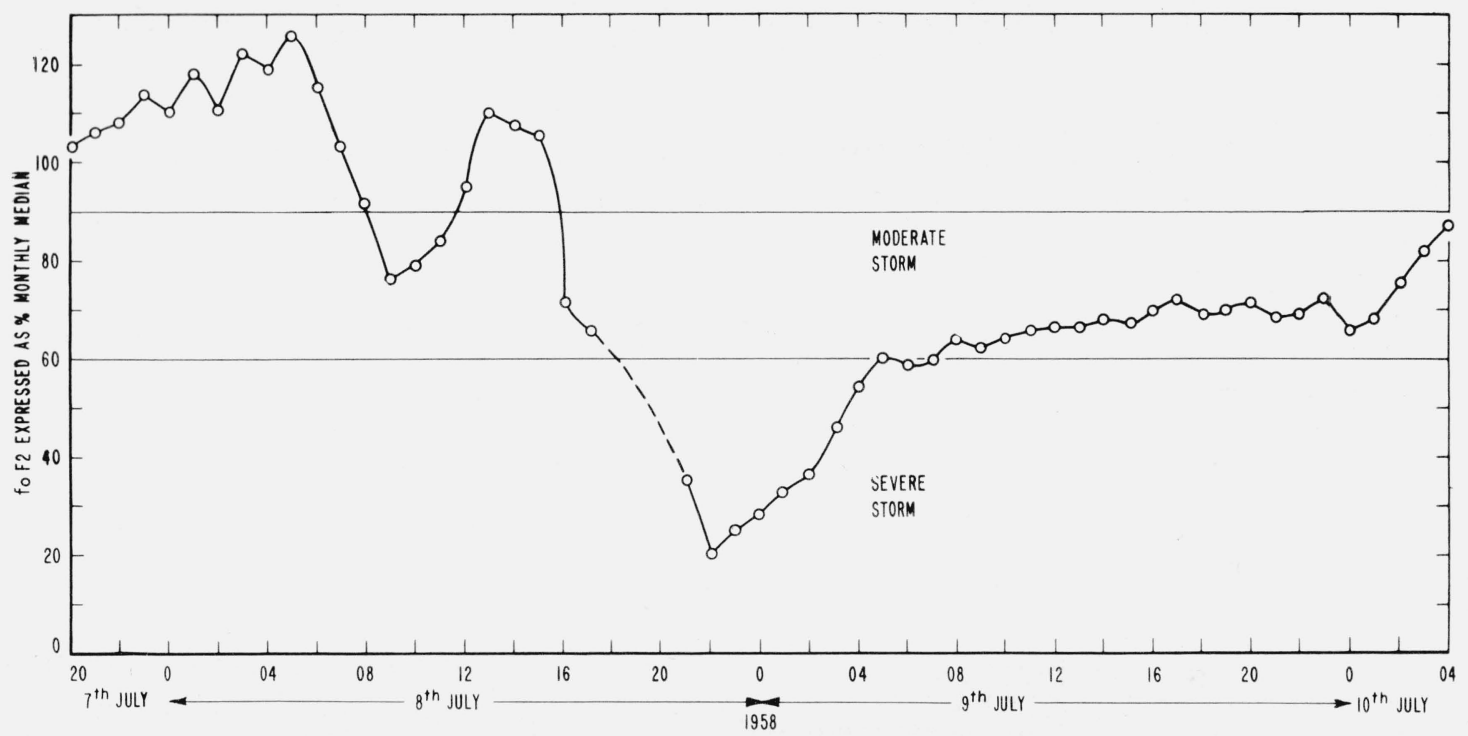

Figure 1. Ionospheric storm on 7 th, 8th, 9th, and 10th, of July 1958, readings at slough. Indicates discontinuity in readings.

It is, of course, rather arbitrary to define the start and finish of a fairly widespread storm by means of soundings taken at a single station. Comparison of Slough and Inverness data showed that the broad features of the behaviour of $f_{0} F 2$ were the same at these stations, but that the smaller features of the plotted records were not identical. Measurements from other European sounding stations had not been published when the analysis was undertaken. Since the local times at points of reflection for most of the tasks analyzed did not differ by more than about two hours from the time at Slough, the behaviour of fo F2 at Slough was probably reasonably representative of conditions over the area concerned.

\section{Dispersion Factor}

As already explained, the computer calculates a $\mathrm{BPE}$ of the position of the target transmitter for each set of bearings fed to it. The observed bearings are then compared with the bearings which would pass through the BPE, and an angular residual formed for each contributing DF station. Each residual is squared and divided by the Brooke variance of the bearing. The dispersion factor is then the sum of the resulting numbers for all the bearings contributing to the fix. The factor is calculated by the computer to one decimal place.

Simple statistical theory suggests that the dispersion factors for $n$ stations should have a $\chi^{2}$ distribution with $(n-2)$ degrees of freedom. Details of this distribution may be found in most textbooks on statistics, e.g.. Yule and Kendall, Introduction to the Theory of Statistics, chapter 20. The mean value of the dispersion factors should be $(n-2)$ and the variance about the mean should be $2(n-2)$.

These results depend on the assumptions usually made in DF analysis, that bearing errors are normally distributed about zero mean and that the errors in bearings taken from different stations on the same task are not correlated. It is known, however, that none of these conditions is strictly satisfied in practice. In particular, it seems that the leptokurtic distributions of bearing errors which occur in practice will lead to a variance rather greater than $2(n-2)$. It is difficult to estimate the effect on the dispersion factors of small systematic errors in bearings and of correlation between bearing errors. These points have not been considered in detail here, since we are concerned more with a comparison of factors under different conditions than with their absolute values.

The dispersion factor will be denoted by $\Sigma_{0}$ following the notation used by Beale and others. No convenient name or symbol exists for the quantity $\left\{\Sigma_{0}-(n-2)\right\}$; the symbol $\Sigma_{n}$ will be used in this paper. We except from simple theory that $\Sigma_{n}$ will be zero on average, positive under adverse conditions and negative under favourable conditions. It will be shown later that, in practice, the average value of $\Sigma_{n}$ for a given day tends to be positive.

\section{Comparison of Calm and Stormy Days}

A period of six months, from July to December 1958 inclusive, was selected for study. According to the B.B.C. reports, severe storms occurred on 15 days in this period and there were 49 days with moderate storms.

It was decided to compare values of $\Sigma_{n}$ on days of severe storms with values on samples of calm days. Saturdays and Sundays were not used in either sample because computer results for tasks on these days had not been calculated. There then remained 12 days of severe storms for study. Four calm days were chosen, one each in July, September, October, and November, thus giving a reasonable distribution throughout the period. Days on which magnetic storms or Dellinger fades occurred were not selected for this sample, but otherwise the days were chosen at random. 
The results are set out in table 1 below. The average value of $\Sigma_{n}$ for all stormy days is +1.27 and is significantly greater than the value of +0.57 on calm days.

It is clear from table 1 that propagation conditions were worse from the point of view of DF accuracy on days of severe storms than on calm days, as expected. The question next arises as to whether this effect applies to all frequencies, or is more marked in a particular part of the HF spectrum. Table 2 shows the value of $\Sigma_{n}$ for $1 \mathrm{Mc} / \mathrm{s}$ intervals for the same sample of calm and stormy days. The difference between the results is more marked on the first and second frequency bands (i.e., up to $10 \mathrm{Mc} / \mathrm{s}$ ) than on the third band, but all are affected to some extent.

In order to investigate further the effect of storms on accuracy, it was decided to use Slough measurements of fo $F_{2}$ to define periods of moderate and severe storms as described earlier. The period considered was extended to June 1959. Table 3 shows values of $\Sigma_{n}$ during the moderate and severe phases of the storms considered.

TABLE 1. Comparison of calm days with days of severe storms

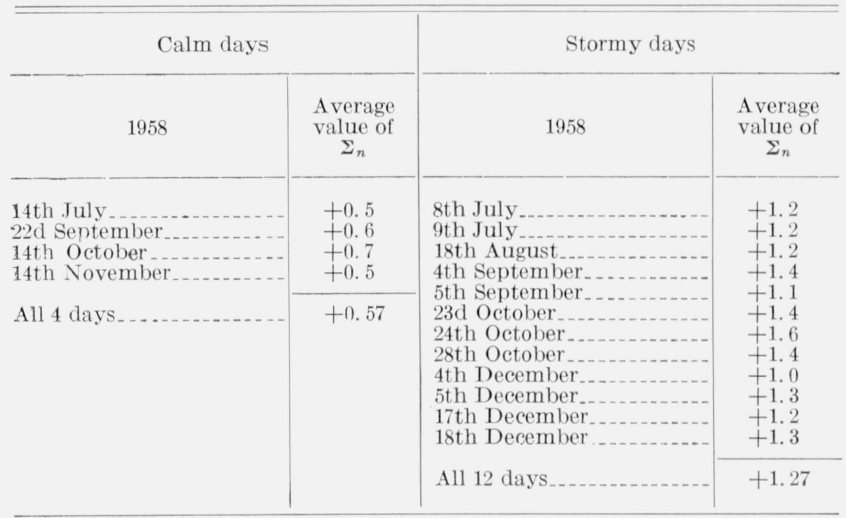

\begin{tabular}{|c|c|c|}
\hline \multirow{2}{*}{ Frequency } & Calm days & Stormy days \\
\hline & $\begin{array}{l}\text { A verage value } \\
\text { of } \Sigma_{n}\end{array}$ & $\begin{array}{l}\text { A verage value } \\
\text { of } \Sigma_{n}\end{array}$ \\
\hline $\begin{array}{l}\mathrm{Mc} / \mathrm{s} \\
1-2 \\
2-3 \\
3-4 \\
4-5 \\
5-6\end{array}$ & $\begin{array}{l}+0.3 \\
+1.5 \\
+1.2 \\
+0.2\end{array}$ & $\begin{array}{r}+2.7 \\
+1.9 \\
+2.0 \\
+1.6 \\
+1.6\end{array}$ \\
\hline $1-6$ & +.95 & +1.72 \\
\hline $\begin{array}{l}6-7 \\
7-8 \\
8-9 \\
9-10\end{array}$ & $\begin{array}{r}+.8 \\
+.4 \\
+.2 \\
.0\end{array}$ & $\begin{array}{r}+1.5 \\
+1.4 \\
+1.3 \\
+1.4\end{array}$ \\
\hline $6-10$ & +.47 & +1.41 \\
\hline $\begin{array}{l}10-11 \\
11-12 \\
12-13 \\
13-14 \\
14-15 \\
>15\end{array}$ & $\begin{array}{r}-.3 \\
.0 \\
+.3 \\
+.2 \\
+.6 \\
+1.0\end{array}$ & $\begin{array}{r}+0.2 \\
+1.0 \\
0.0 \\
+.4 \\
-.1 \\
+.9\end{array}$ \\
\hline$>10$ & +0.17 & +.48 \\
\hline
\end{tabular}

TABLE 3. $\quad \Sigma_{n}$ for moderate and severe phases of storms

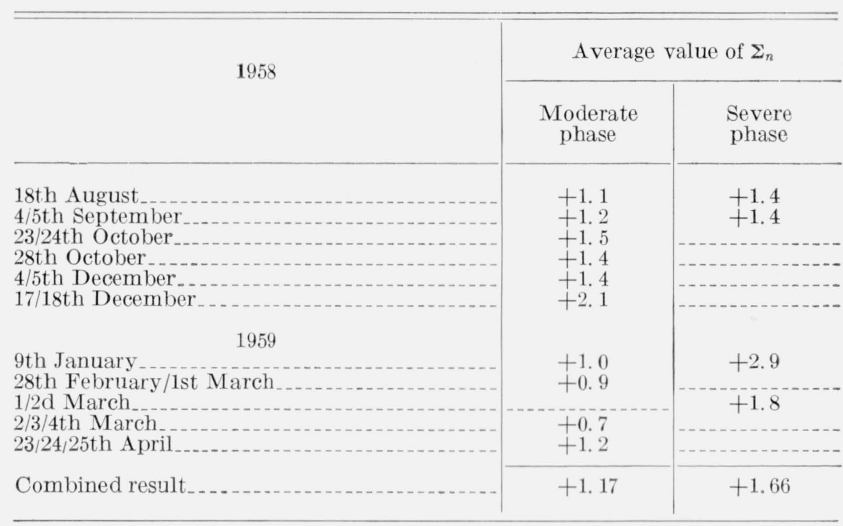

The storm on 8th and 9th July 1958 had to be omitted because the relevant logs had been destroyed.

The average value of $\Sigma_{n}$ shows the expected increase under increasingly stormy conditions.

It should be noted that several dispersion factors were affected by the rejection of bearings showing large departures from the BPE. Either machine rejection or operator rejection may be applied to the results calculated by the computer; the criteria for rejection will not be described in detail here, but depend in both cases on an examination of the dispersion factor. Rejection of a bearing which is obviously "wild" normally leads to a considerable reduction in this factor.

An examination of computer results showed that a higher percentage of bearings were rejected on stormy days than on calm days. On days of severe storms about 3.9 percent of all bearings were rejected, whereas on the calm days examined only 1.2 percent were rejected. This result is to be expected in view of the increased size of the dispersion factors on stormy days. The greater percentage of rejections on stormy days tends to reduce the difference between the average value of $\Sigma_{n}$ on calm and stormy days; hence the difference would be even more marked than in table 1 if the same percentage of bearings had been rejected on both types of day.

A sort was carried out to decide whether bearings taken by any particular DF operators gave rise on average to abnormally large or small contributions to the dispersion factors. Results were inconclusive.

\section{Dependence on Mode of Propagation}

Individual tasks on two calm and two stormy days were examined with a view to determining the probable mode of propagation.

The range from the $\mathrm{BPE}$ for the transmitter to London was taken to be a reasonable approximation to the true distance of the transmitter from the DF station. The maximum usable frequency (MUF) for the same path was derived for the $E, F 1$, and $F 2$ layers from the U.S. Central Radio Propagation Laboratory (CRPL) predictions. These frequencies were then compared with the known signal fre- 
quency, and possible modes of propagation noted. By suitable division of the path it was possible to decide whether multiple-hop modes were likely.

The tasks were then sorted into two groups depending on whether or not propagation was via the F2 layer. The sort was necessarily approximate, since neither the predicted frequencies nor the distances used were free from error. However, the assignment of mode of propagation was expected to be accurate enough to reveal any large difference in DF accuracy dependent on the layer involved.

Results of the sort are shown in table $4 . \quad \Sigma_{n}$ was again used as a measure of the scatter of the bearings. From the table it appears that direction finding on signals propagated via the $E$ or $F 1$ layers is less affected by ionospheric storms than direction finding on signals propagated via the F2 layer.

TABLE 4. Dependence of $\Sigma_{\mathrm{n}}$ on probable mode of propagation

\begin{tabular}{l|c|c}
\hline \hline & \multicolumn{2}{|c}{ Average values of $\Sigma_{n}$} \\
\cline { 2 - 3 } & $\begin{array}{c}\text { Two calm days } \\
\text { E and F1 }\end{array}$ & $\begin{array}{c}\text { Two stormy days } \\
+0.7\end{array}$ \\
\hline F2 & $\begin{array}{c}+0.5 \\
+.6\end{array}$ \\
\hline
\end{tabular}

\section{Effect of Storms on Systematic Errors}

The errors of bearings taken on targets of known position were calculated and averaged for each DF station for three calm days and three stormy days. On the calm days, the average systematic error was $+0.6^{\circ}$; on the stormy days it was $+0.1^{\circ}$.
The nature of systematic errors is not completely understood, and it is therefore not possible to draw any conclusions from the reduction when conditions are stormy. Each systematic error probably represents a combination of effects due to instrumental errors, site errors not removed by calibration and ionospheric effects.

\section{Conclusions}

The investigation has established that DF accuracy deteriorates during ionospheric storms and is worst during severe storms. The effect of a storm may extend over the whole HF band, but is more marked below $10 \mathrm{Mc} / \mathrm{s}$ than above. The main effect seems to be on signals propagated via the $F 2$ layer. Systematic errors during storms generally become less positive.

The results imply that the Brooke variances should be increased during storm periods; such an increase would lead to rather larger probability rectangles on fixes taken in these periods.

The present investigation demonstrates the advantages of adopting an objective classification system for bearings and of using an electronic computer to reduce DF data on a uniform basis and in a form suitable for analysis.

The author thanks Miss A. Partridge and Mr. R. M. Rampling who carried out most of the analysis and who made many valuable suggestions.

This paper is published by permission of the Director, Government Communications Headquarters, Cheltenham, England.

(Paper 65D3-122) 